\title{
ON THE HOMOTOPY CLASSIFICATION OF THE EXTENSIONS OF A FIXED MAP
}

BY

\author{
W. D. BARCUS AND M. G. BARRATT
}

1. Introduction. In considering the homotopy classification of the maps of a CW complex into any topological space $X$, we are led to the problem of enumerating the homotopy classes of extensions of a given map $u: K \rightarrow X$ over a larger complex $L \supset K$. We examine this for the case in which $L-K$ consists only of disjoint cells, for maps and homotopies relative to a base point $k_{0} \in K$.

For a given map $u:\left(K, k_{0}\right) \rightarrow\left(X, x_{0}\right)$, we define in $\S 2$ for each $\alpha \in \pi_{q}\left(K, k_{0}\right)$ a homomorphism

$$
\alpha_{u}: \pi_{1}(F, u) \rightarrow \pi_{q+1}\left(X, x_{0}\right)
$$

where $\mathcal{F}$ is the function space of maps $\left(K, k_{0}\right) \rightarrow\left(X, x_{0}\right)$. If $L=K \cup e^{q+1}$ is formed by attaching the cell $e^{q+1}$ by a map in the class $\alpha$, and if $u$ extends over $L$, then we prove that the homotopy classes (rel $k_{0}$ ) of extensions are in 1-1 correspondence with the cokernel of $\alpha_{u}$. This may easily be generalized to a complex $L=K \cup\left\{e^{q_{i}+1}\right\}$ such that the $e^{q_{i}+1}$ are disjoint.

The difficulty lies in computing $\alpha_{u}$, even when the group $\pi_{1}(\mathfrak{F}, u)$ is known. We show how $\alpha_{u}$ can be computed when $K$ is a cluster of spheres: the result is given in terms of $\alpha$, its Hopf invariants (including the higher Hopf invariants in the sense of Hilton [3]), the homotopy groups of $X$, and the operations of composition, suspension, and formation of Whitehead products. This covers, for example, the case when $L$ is a sphere bundle over a sphere with a cross-section, such as the product of two spheres.

In $\$ 7$ we give applications of the theory to two other problems; the more important of these is a formula for expanding a Whitehead product of the form $[\alpha \circ \gamma, \beta]$. It should be noted that the Whitehead product we use (\$4) differs from that defined by J. H. C. Whitehead by a sign.

2. Homotopy groups of function spaces. Let $K$ be a CW complex. The function space $X^{K}$ of maps (=continuous functions) is given the compactopen topology. Then the natural function $\theta: X^{(K \times T)} \rightarrow\left(X^{K}\right)^{T}$, given by

$$
(\theta f)(t)(k)=f(k, t), \quad k \in K, t \in T,
$$

is a homeomorphism if $T$ is a CW complex such that $K \times T$, given the product topology, is also a CW complex (the proof is elementary; cf. [2] and [9] for other cases in which $\theta$ is a homeomorphism). Notice that if $I$ is the unit

Received by the editors October 20, 1956. 
interval, then $K \times I$ is always a CW complex. It is convenient to identify $X^{K \times I}$ and $\left(X^{K}\right)^{I}$ by means of $\theta$.

Notation. A fixed base point will always be chosen in each space, and denoted by a subscript 0 : thus, $k_{0} \in K, x_{0} \in X$. The only exception is that $0=(0, \cdots, 0)$ will be the base point in $I^{n}$; the base point in $K \times I^{n}$ will be $\left(k_{0}, 0\right)$. The function space, with the compact-open topology, of maps $\left(K, k_{0}\right)$ $\rightarrow\left(X, x_{0}\right)$ will in the future be denoted by $X^{K}$; no ambiguity will arise, since no further reference will be made to the space of maps $K \rightarrow X$. The domain space $K$ will always be assumed to be a CW complex, $k_{0}$ a vertex.

Let $u:\left(K, k_{0}\right) \rightarrow\left(X, x_{0}\right)$ be a map; it follows from the first paragraph of this section that we may equally well represent elements of $\pi_{1}\left(X^{K}, u\right)$ as homotopy classes of maps

$$
\hat{F}: I \rightarrow X^{K} \text { such that } \hat{F}(0)=u=\hat{F}(1),
$$

or

$F:\left(K \times I, k_{0} \times I\right) \rightarrow\left(X, x_{0}\right)$ such that $F(k, 0)=u(k)=F(k, 1), \quad k \in K$. Therefore a map $g:\left(Q, q_{0}\right) \rightarrow\left(K, k_{0}\right)$ induces a homomorphism

$$
g^{*}: \pi_{1}\left(X^{K}, u\right) \rightarrow \pi_{1}\left(X^{Q}, u g\right)
$$

by $g^{*}\{F\}=\{F(g \times 1)\}$, where 1 is the identity map of $I$ and

$$
g \times 1:\left(Q \times I, q_{0} \times I\right) \rightarrow\left(K \times I, k_{0} \times I\right)
$$

is the product map.

Now a path $\hat{L}$ in $X^{K}$ from $u_{0}$ to $u_{1}$ is equivalent to a homotopy $L:\left(K \times I, k_{0} \times I\right) \rightarrow\left(X, x_{0}\right)$ from $u_{0}$ to $u_{1}$; the path $\hat{L}$ defines an isomorphism in the usual way from the homotopy groups based at $u_{1}$ to those based at $u_{0}$ : we write for this

$$
L_{\sharp}: \pi_{1}\left(X^{K}, u_{1}\right) \rightarrow \pi_{1}\left(X^{K}, u_{0}\right) .
$$

Lemma (2.2). $g^{*} L \#=(L(g \times 1)) g^{*}: \pi_{1}\left(X^{K}, u_{1}\right) \rightarrow \pi_{1}\left(X^{Q}, u_{0} g\right)$.

Let $g_{0}, g_{1}:\left(Q, q_{0}\right) \rightarrow\left(K, k_{0}\right)$, and let $G:\left(Q \times I, q_{0} \times I\right) \rightarrow\left(K, k_{0}\right)$ be a homotopy from $g_{0}$ to $g_{1}$. Then

Lemma (2.3). $g_{0}^{*}=(u G) z_{1}^{*}: \pi_{1}\left(X^{K}, u\right) \rightarrow \pi_{1}\left(X^{Q}, u g_{0}\right)$.

The proofs of these elementary lemmas are omitted; it is easy to deduce from them

Corollary (2.4). If $g$ is a homotopy equivalence, then $g^{*}$ is an isomorphism.

Suppose now that $\left(Q, q_{0}\right)=\left(S^{q}, s_{0}\right)$, where we consider $S^{q}=s_{0} \cup e^{q}$ as a $\mathrm{CW}$ complex with a characteristic map

$$
i^{q}:\left(I^{q}, \dot{I}^{q}\right) \rightarrow\left(S^{q}, s_{0}\right)
$$


which is a homeomorphism of $I^{q}-\dot{I}^{q}$ onto $e^{q}$ of degree +1 . Let $v$ : $\left(S^{q}, s_{0}\right)$ $\rightarrow\left(X, x_{0}\right)$, and define $v^{b}:\left(S^{q} \times I, s_{0} \times I\right) \rightarrow\left(X, x_{0}\right)$ by $v^{b}(s, t)=v(s)$, for $s \in S^{q}$, $t \in I$. We then define

$$
v_{\natural}: \pi_{1}\left(X^{s^{q}}, v\right) \rightarrow \pi_{q+1}\left(X, x_{0}\right)
$$

as follows: for $\{F\} \in \pi_{1}\left(X^{s^{q}}, v\right), v_{\natural}\{F\}$ is the value of the separation element $\left.{ }^{1}\right) d\left(F, v^{b}\right)$ on the cell $\left(e^{q} \times e^{1}, s_{0} \times 0\right)$ with the product orientation, where $e^{1}=I-\dot{I}$. It is readily verified that

Lemma (2.6). $v_{\natural}$ is an isomorphism; and if $M$ is a homotopy from $v$ to $v^{\prime}$, then $v_{\sharp}^{\prime}=v_{\mathrm{g}} M_{\mathrm{H}}$.

Now let $g_{0}, g_{1}:\left(S^{q}, s_{0}\right) \rightarrow\left(K, k_{0}\right)$, let $G$ be a homotopy from $g_{0}$ to $g_{1}$, and let $u \in X^{K}$. Then

$$
\begin{aligned}
\left(u g_{0}\right) \backsim g_{0}^{*} & =\left(u g_{0}\right) \mathfrak{\natural}(u G)_{\sharp g_{1}^{*}}^{*} & & \text { by }(2.3), \\
& =\left(u g_{1}\right) \text { घg} g_{1}^{*} & & \text { by }(2.6) .
\end{aligned}
$$

Hence the homomorphism $(u g)_{\natural} g^{*}$ depends only on the homotopy class $\alpha \in \pi_{q}\left(K, k_{0}\right)$ of $g$, and we may define

$$
\alpha_{u}=(u g) \mathrm{g} g^{*}: \pi_{1}\left(X^{K}, u\right) \rightarrow \pi_{q+1}\left(X, x_{0}\right) .
$$

Lemma (2.8). If $L$ is a homotopy from $u_{0}$ to $u_{1}$, then $\alpha_{u_{1}}=\alpha_{u_{0}} L_{\# \text { f }}$.

The lemma follows from (2.2) and (2.6).

3 . The classification theorems. We now explain the use of $\alpha_{u}$ in homotopy classification. First, let $L=K \cup e^{q+1}$, where $e^{q+1}$ has an attaching map $g:\left(S^{q}, s_{0}\right) \rightarrow\left(K, k_{0}\right)$ in a homotopy class $\alpha \in \pi_{q}\left(K, k_{0}\right)$. Then a map $u:\left(K, k_{0}\right)$ $\rightarrow\left(X, x_{0}\right)$ has an extension to $\left(L, k_{0}\right)$ if and only if

$$
u_{*} \alpha=0 \text {. }
$$

If this is satisfied, let $f_{0}, f_{1}$ be two extensions of $u$ such that there is a homotopy $\bar{H}:\left(L \times I, k_{0} \times I\right) \rightarrow\left(X, x_{0}\right)$ from $f_{0}$ to $f_{1}$. Then $H=\bar{H} \mid\left(K \times I, k_{0} \times I\right)$ determines an element $\{H\} \in \pi_{1}\left(X^{K}, u\right)$ : we shall prove

Lemma (3.2). The value of the separation element $d\left(f_{1}, f_{0}\right)$ on the cell $\left(e^{q+1}, k_{0}\right)$ is $\alpha_{u}\{H\} \in \pi_{q+1}\left(X, x_{0}\right)$.

From the lemma we deduce

Theorem (3.3). Let $u:\left(K, k_{0}\right) \rightarrow\left(X, x_{0}\right)$ extend to $\left(L, k_{0}\right)$. Then the homotopy classes rel $k_{0}$ of extensions are in 1-1 correspondence with the elements of the cokernel of $\alpha_{u}$, i.e. of $\pi_{q+1}\left(X, x_{0}\right) / \alpha_{u} \pi_{1}\left(X^{K}, u\right)$.

The lemma leads in fact to a more general result: let $L=K \cup\left\{e^{q_{i+1}}\right\}$, where the cells $e^{q_{i}+1}$ are disjoint, and each possesses an attaching map

() Cf. Appendix. 
$g_{i}:\left(S^{q i}, s_{0}\right) \rightarrow\left(K, k_{0}\right)$ in a class $\alpha_{i} \in \pi_{q_{i}}\left(K, k_{0}\right)$. Set $C(L, K)=\sum \pi_{q_{i}+1}\left(X, x_{0}\right)$, the strong sum, where the homotopy groups are indexed by the cells of $L-K$; a map $u:\left(K, k_{0}\right) \rightarrow\left(X, x_{0}\right)$ extends to $\left(L, k_{0}\right)$ if and only if $u_{*} \alpha_{i}=0$ for all $i$. Then the homomorphisms $\left(\alpha_{i}\right)_{u}$ together define

$$
\alpha_{u}: \pi_{1}\left(X^{K}, u\right) \rightarrow C(L, K)
$$

such that the coordinate of $\alpha_{u}(\xi)$ in $\pi_{q_{i+1}}\left(X, x_{0}\right)$ is $\left(\alpha_{i}\right)_{u}(\xi)$.

Theorem (3.4). Let $u:\left(K, k_{0}\right) \rightarrow\left(X, x_{0}\right)$ extend to $\left(L, k_{0}\right)$. Then the homotopy classes rel $k_{0}$ of extensions are in 1-1 correspondence with the elements of the cokernel of $\alpha_{u}$, i.e. with the cosets $C(L, K) / \alpha_{u} \pi_{1}\left(X^{K}, u\right)$.

We now prove (3.2)-(3.4); we first need an elementary lemma which will be used again later.

Let $P$ be a finite CW complex on $I^{n}$ such that $0=(0, \cdots, 0)$ is a vertex. Let $\left\{\sigma^{n}\right\}$ be the set of $n$-cells of $P$, and let the orientation of each, given by the chosen characteristic map $c_{\sigma}:\left(I^{n}, \dot{I}^{n}, 0\right) \rightarrow\left(\bar{\sigma}^{n}, \dot{\sigma}^{n}, p_{\sigma}\right)$, agree with the orientation induced by inclusion in $I^{n}$. For each $\sigma$ let $T_{\sigma}:(I, 0,1) \rightarrow\left(P, 0, p_{0}\right)$ be a path in $P$. Suppose that $h^{\prime}, h:(P, 0) \rightarrow\left(X, x_{0}\right)$ agree on $P^{n-1}$. Then the separation element $d\left(h^{\prime}, h\right)$ on $\left(\sigma, p_{\sigma}\right)$ has a value $\delta_{\sigma} \in \pi_{n}\left(X, h p_{\sigma}\right)$. Treating $I^{n}$ as a $C W$ complex with just one $n$-cell in the usual way, we also have a separation element $d\left(h^{\prime}, h\right)$ on $\left(I^{n}, 0\right)$ with a value $\delta \in \pi_{n}\left(X, x_{0}\right)$.

Lemma (3.5). $\delta=\sum\left(h T_{\sigma}\right) \# \delta_{\sigma}$, where \# denotes the operation of the path on the homotopy group, and the summation is over all $\sigma \in\left\{\sigma^{n}\right\}$.

Since all paths $T_{\sigma}$ for a given $p_{\sigma}$ are homotopic in $I^{n},\left(h T_{\sigma}\right) \#$ does not depend on the choice of $T_{\sigma}$. Notice that an equivalent result holds with $I^{n}$ replaced by a sphere $S^{n}$, taking $i^{n}$ as the characteristic map of the cell $e^{n}$ $=S^{n}-s_{0}$.

The proof of this lemma is omitted.

Proof of (3.2). We identify $\left(S^{q}, s_{0}\right)$ with $\left(\dot{I}^{q+1}, 0\right)$, and write $j=j^{q}:\left(S^{q}, s_{0}\right)$ $\rightarrow\left(\dot{I}^{q+1}, 0\right)$ for the identity map. We first show that the triple $\left(L, K, k_{0}\right)$ $=\left(I^{q+1}, S^{q}, 0\right)$ is a universal example. Let the cell $e^{q+1}$ in $L=K \cup e^{q+1}$ have characteristic map $\bar{g}:\left(I^{q+1}, S^{q}, 0\right) \rightarrow\left(L, K, k_{0}\right)$, and attaching map $g=\bar{g} \mid\left(S^{q}, 0\right)$. Let $e_{0}^{q+1}=I^{q+1}-S^{q}$ have characteristic and attaching maps $\bar{j}, j$, the identity maps; and using the notation of (3.2), set $f_{1}^{\prime}=f_{1} \bar{g}, f_{0}^{\prime}=f_{0} \bar{g}, \bar{H}^{\prime}=\bar{H}(g \times 1)$, $H^{\prime}=H(g \times 1), u^{\prime}=u g$. Suppose that (3.2) holds for the universal example, so that $d\left(f_{1}^{\prime}, f_{0}^{\prime}\right)=\iota_{u^{\prime}}\left\{H^{\prime}\right\}$, where $\iota$ is the class of $j$, and the separation element is evaluated on $\left(e_{0}^{q+1}, 0\right)$. Then if $d\left(f_{1}, f_{0}\right)$ is evaluated on $\left(e^{q+1}, k_{0}\right)$ we have

$$
\begin{aligned}
& d\left(f_{1}, f_{0}\right)=d\left(f_{1}^{\prime}, f_{0}^{\prime}\right)=\iota u^{\prime}\left\{H^{\prime}\right\}=u \prime^{\prime} j^{*}\left\{H^{\prime}\right\} \\
& =u \natural^{\prime}\left\{H^{\prime}\right\}=(u g) \text { ต }\{H(g \times 1)\} \\
& =(u g) g^{*}\{H\}=\alpha_{u}\{H\} \text {, }
\end{aligned}
$$

from the definitions. 
We now prove (3.2) for the universal example by means of an explicit construction. The separation element $d\left(f_{1}, f_{0}\right)$ on $\left(e_{0}^{q+1}, 0\right)$ is represented by the map $E:\left(\left(I^{q+1} \times I\right)^{\cdot}, 0\right) \rightarrow\left(X, x_{0}\right)$ given by

$$
E(p, t)=\left\{\begin{array}{cl}
f_{1}(p), & t=1 \\
f_{1}(p)=f_{0}(p), & 0<t<1 \\
f_{0}(p), & t=0 .
\end{array}\right\}(p, t) \in\left(I^{q+1} \times I\right)^{q} .
$$

Take a cellular decomposition of $\dot{I}^{q+2}=\left(I^{q+1} \times I\right)^{\cdot}$ such that $\dot{I}^{q+1}=0 \cup e^{q}$; $I^{q+1}=\dot{I}^{q+1} \cup e^{q+1} ; I=0 \cup 1 \cup e^{1}$. Thus

$$
\dot{I}^{q+2}=\left(\dot{I}^{q+1} \times \dot{I} \cup 0 \times I\right) \cup\left(e^{q+1} \times 0\right) \cup\left(e^{q+1} \times 1\right) \cup\left(e^{q} \times e^{1}\right) .
$$

Now $\bar{H}$ agrees with $E$ on the $q$-section of $\dot{I}^{q+2}$, and also on the cells $e^{q+1} \times 0$, $e^{q+1} \times 1$. Hence, by using Lemma (3.5) for a sphere, and noting that the orientation of $e^{q} \times e^{1}$ is opposite to that induced by inclusion in $\dot{I}^{q+2}$, the separation element $d\left(\bar{H} \mid\left(I^{q+1} \times I\right)^{\circ}, E\right)$ on $\left(\dot{I}^{q+2}, 0\right)$ is equal to minus the element $d\left(\bar{H}\left|\dot{I}^{q+1} \times I, E\right| \dot{I}^{q+1} \times I\right)$ on $\left(e^{q} \times e^{1}, 0\right)$. But maps of $\left(\dot{I}^{q+2}, 0\right)$ into $\left(X, x_{0}\right)$ determine elements of $\pi_{q+1}\left(X, x_{0}\right)$, so that the former separation element is

$$
\left\{\bar{H} \mid\left(I^{a+1} \times I\right) \cdot\right\}-\{E\}=0-\{E\}=-\{E\} ;
$$

and since $\bar{H}\left|\dot{I}^{q+1} \times I=H, E\right| \dot{I}^{q+1} \times I=u^{b}$, the latter separation element is

$$
\begin{aligned}
d\left(H, u^{b}\right)\left(e^{q} \times e^{1}, 0\right) & =u_{\natural}\{H\} \text { by definition, } \\
& =u_{\xi} j^{*}\{H\}=\iota_{u}\{H\} .
\end{aligned}
$$

Hence $d\left(f_{1}, f_{0}\right)\left(I^{q+1}, 0\right)=\iota_{u}\{H\}$, which proves (3.2) for the universal example.

Proof of (3.3). The homotopy classes rel $K$ of extensions of $u$ are in 1-1 correspondence with the elements of $\pi_{q+1}\left(X, x_{0}\right)$; they may be distinguished by the separation elements of representative maps. Let $f_{0}, f_{1}$ be two extensions of $u$ for which there is a homotopy $\bar{H}$ rel $k_{0}$ from $f_{0}$ to $f_{1}$. Then by (3.2), the separation element on $\left(e^{q+1}, k_{0}\right)$ is contained in $\alpha_{u} \pi_{1}\left(X^{K}, u\right)$. Conversely, if $f_{0}, f_{1}$ are two extensions of $u$ such that $d\left(f_{1}, f_{0}\right)=\alpha_{u}\{H\}$, with $H:(K \times I$, $\left.k_{0} \times I\right) \rightarrow\left(X, x_{0}\right)$, let $\bar{H}$ be an extension of $H$ to $L \times I$ such that $\bar{H}(p, 0)=f_{0}(p)$, $p \in L$, and define $f_{1}^{\prime}:\left(L, k_{0}\right) \rightarrow\left(X, x_{0}\right)$ by $f_{1}^{\prime}(p)=\bar{H}(p, 1)$. Then by (3.2), $d\left(f_{1}^{\prime}, f_{0}\right)=\alpha_{u}\{H\}=d\left(f_{1}, f_{0}\right)$. Hence $d\left(f_{1}, f_{1}^{\prime}\right)=0$, and $f_{1} \simeq f_{1}^{\prime}$ rel $K$. Since $f_{1}^{\prime} \simeq f_{0}$ rel $k_{0}, f_{1} \simeq f_{0}$ rel $k_{0}$.

Proof of (3.4). If $L=K \cup\left\{e^{q_{i}+1}\right\}$ is formed by attaching a set of cells to $K$, we may alter $K$ within homotopy type so that the base point $k_{0}$ lies on the boundary of each cell; this does not change the group $\pi_{1}\left(X^{K}, u\right)$ by more than an isomorphism. Then, if $u$ extends to two maps $f_{0}, f_{1}:\left(L, k_{0}\right) \rightarrow\left(X, x_{0}\right)$, the maps determine an element $d\left(f_{1}, f_{0}\right) \in C(L, K)$ such that the coordinate of $d\left(f_{1}, f_{0}\right)$ in $\pi_{\mathbf{q}_{i+1}}\left(X, x_{0}\right)$ is $d\left(f_{1}, f_{0}\right)\left(e^{q_{i}+1}, k_{0}\right)$ (which may be defined in the sub- 
complex $\left.K \cup e^{q_{i+1}}\right)$. Then it is easy to show from Lemma (3.2) by the method used in the proof of (3.3) that $f_{0}$ and $f_{1}$ are homotopic if and only if there exists $\{H\} \in \pi_{1}\left(X^{K}, u\right)$ such that $d\left(f_{1}, f_{0}\right)\left(e^{q_{i}+1}, k_{0}\right)=\left(\alpha_{i}\right)_{u}\{H\}$ for all $i$. The theorem then follows at once.

An alternative proof of the above two theorems can be obtained by considering homotopy sequences of the fibering $X^{L} \rightarrow X^{K}$ induced by the inclusion $K \subset L$.

4. The addition, product, and composition theorems. In this section we give three theorems which are useful in the computation of the homomorphism $\alpha_{u}$.

Let $\alpha, \beta \in \pi_{q}\left(K, k_{0}\right), u \in X^{K}, \xi \in \pi_{1}\left(X^{K}, u\right)$; and let $\cdot$ denote the operation of $\pi_{1}$ on $\pi_{r}$.

Theorem (4.1) (Addition Theorem). If $q>1$,

$$
(\alpha+\beta)_{u}(\xi)=\alpha_{u}(\xi)+\beta_{u}(\xi) ;
$$

if $q=1$ (so that $u_{*} \alpha \in \pi_{1}\left(X, x_{0}\right)$ ), then

$$
(\alpha+\beta)_{u}(\xi)=\alpha_{u}(\xi)+\left(u_{*} \alpha\right) \cdot \beta_{u}(\xi) .
$$

Thus if $q>1$, the transformation $(\alpha, \xi) \rightarrow \alpha_{u}(\xi)$ is a pairing of $\pi_{q}\left(K, k_{0}\right)$ and $\pi_{1}\left(X^{K}, u\right)$ to $\pi_{q+1}\left(X, x_{0}\right)$; if $q=1$, the transformation might be called a crossed pairing. We shall prove the theorem later, by means of an explicit construction.

Now let $\gamma \in \pi_{m}\left(K, k_{0}\right), \delta \in \pi_{n}\left(K, k_{0}\right)$ be represented by maps $f:\left(I^{m}, \dot{I}^{m}\right)$ $\rightarrow\left(K, k_{0}\right)$ and $g:\left(I^{n}, \dot{I}^{n}\right) \rightarrow\left(K, k_{0}\right)$ respectively. Then the Whitehead product $[\gamma, \delta]$ is defined to be the class of the map $p:\left(\dot{I}^{m+n}, 0\right)=\left(I^{m} \times \dot{I}^{n} \cup \dot{I}^{m} \times I^{n}, 0\right)$ $\rightarrow\left(K, k_{0}\right)$ given by

$$
\begin{aligned}
p(s, t)= & f(s), s \in I^{m}, t \in \dot{I}^{n} \\
& g(t), s \in \dot{I}^{m}, t \in I^{n} .
\end{aligned}
$$

Notice that because of our orientation conventions (cf. Appendix), $[\gamma, \delta]$ is not the same as that defined by J. H. C. Whitehead in [8]; we write the latter, defined by using homology orientations, as $[\gamma, \delta]^{\prime}$. The relation is easily seen to be $[\gamma, \delta]=(-1)^{m+n-1}[\gamma, \delta]^{\prime}$.

Let $u \in X^{K}, \xi \in \pi_{1}\left(X^{K}, u\right)$.

Theorem (4.2) (Product Theorem). $[\gamma, \delta]_{u}(\xi)$ is given by

$$
\begin{array}{ll}
-\left[u_{*} \gamma, \delta_{u}(\xi)\right]+(-1)^{n+1}\left[\gamma_{u}(\xi), u_{*} \delta\right] & \text { if } m, n>1 ; \\
-\left[u_{*} \gamma, \delta_{u}(\xi)\right]+(-1)^{n+1}\left[\gamma_{u}(\xi), u_{*} \gamma \cdot u_{*} \delta\right] & \text { if } m=1, n>1 ; \\
-\left[u_{*} \delta \cdot u_{*} \gamma, \delta_{u}(\xi)\right]+(-1)^{n+1}\left[\gamma_{u}(\xi), u_{*} \delta\right] & \text { if } m>1, n=1 ; \\
-\left[u_{*} \delta \cdot u_{*} \gamma, \delta_{u}(\xi)\right]-(-1)^{n+1} u_{*} \delta \cdot\left[\gamma_{u}(\xi),-\left(u_{*} \gamma \cdot u_{*} \delta\right)\right] & \text { if } m=n=1 .
\end{array}
$$

If we agree to use $\pi_{r}$ for $r>1$ as a trivial group of operators, then (iv) is 
seen to include the other formulae. The proof will be given in $\S 8$.

Two simple consequences of (4.2) are the following:

Corollary (4.3). If $\gamma \in \pi_{1}\left(K, k_{0}\right), \delta \in \pi_{n}\left(K, k_{0}\right), n>1$, then

$$
(\gamma \cdot \delta)_{u}(\xi)=u_{*} \gamma \cdot \delta_{u}(\xi)-\left[\gamma_{u}(\xi), u_{*} \gamma \cdot u_{*} \delta\right] .
$$

This follows from (4.2) (ii) and (4.1), since $\gamma \cdot \delta=[\gamma, \delta]^{\prime}+\delta=(-1)^{n}[\gamma, \delta]$ $+\delta$.

Now let $\alpha=P\left(\delta_{1}, \cdots, \delta_{s}\right)$ be a multiple Whitehead product formed from the ordered set $\delta_{1}, \cdots, \delta_{s}\left(\delta_{i} \in \pi_{n_{i}}\left(K, k_{0}\right), n_{i}>1\right)$ by the insertion of $s-1$ brackets [ ]. Let $P_{i}$ denote the product $P\left(u_{*} \delta_{1}, \cdots,\left(\delta_{i}\right)_{u}(\xi), \cdots, u_{*} \delta_{s}\right)$ formed in the same way, but with $\delta_{j}$ replaced by $u_{*} \delta_{j}$ if $j \neq i$, and $\delta_{i}$ by $\left(\delta_{i}\right)_{u}(\xi)$.

CoROLlaRy (4.4). $\alpha_{u}(\xi)=\sum_{i} \pm P_{i}$, where the signs are determined by $P$ and the integers $n_{i}$.

The proof is by repeated application of (4.2)(i). For example,

$$
\begin{aligned}
{\left[\delta_{1},\left[\delta_{2}, \delta_{3}\right]\right]_{u}(\xi)=} & {\left[u_{*} \delta_{1},\left[u_{*} \delta_{2},\left(\delta_{3}\right)_{u} \xi\right]\right] } \\
& +(-1)^{n_{3}}\left[u_{*} \delta_{1},\left[\left(\delta_{2}\right)_{u} \xi, u_{*} \delta_{3}\right]\right] \\
& +(-1)^{n_{2}+n_{3}-1}\left[\left(\delta_{1}\right)_{u} \xi,\left[u_{*} \delta_{2}, u_{*} \delta_{3}\right]\right] .
\end{aligned}
$$

We now use (4.1) and (4.4) to simplify $\alpha_{u}$ when $\alpha=\beta \circ \phi,\left(\beta \in \pi_{n}\left(K, k_{0}\right)\right.$, $\left.\phi \in \pi_{q}\left(S^{n}, s_{0}\right)\right)$. To express the result we need certain of the higher Hopf invariants of $\phi$ (cf. [3]); the definition of these depends on a choice of basic products $\omega_{i} \in \pi_{r_{i}}\left(S^{n} \bigvee S_{0}^{n}, s_{0}\right), n \geqq 2$, as defined and ordered in [3], with $\omega_{-2}=\iota^{n}, \omega_{-1}=\iota_{0}^{n}$, respectively the generators of $\pi_{n}\left(S^{n} \bigvee S_{0}^{n}, s_{0}\right)$ represented by maps of degree +1 of $S^{n}$ onto $S^{n}$ and $S_{0}^{n}$. Then it is shown in [3] that

$$
\left(\iota^{n}+\iota^{n}\right) \circ \phi=\iota^{n} \circ \phi+\iota_{0}^{n} \circ \phi+\sum_{0}^{\infty} \omega_{i} \circ H_{i}(\phi),
$$

where $H_{i}(\phi) \in \pi_{q}\left(S^{r_{i}}\right)$ is termed a higher Hopf invariant of $\phi$.

For elements $\gamma, \delta$ in the homotopy groups of any space $Y$, define inductively $\sigma_{0}(\gamma, \delta)=[\gamma, \delta], \cdots, \sigma_{p}(\gamma, \delta)=\left[\gamma, \sigma_{p-1}(\gamma, \delta)\right]$. Then it follows from the ordering $\iota^{n}<\iota_{0}^{n}$ chosen above that $\sigma_{p}\left(\iota^{n}, \iota_{0}^{n}\right)$ is a basic product of weight $p+2$ for $p \geqq 0$. If $\sigma_{p}\left(\iota^{n}, \iota_{0}^{n}\right)=\omega_{i_{p}}$, write $B_{p}(\phi)=H_{i_{p}}(\phi)$, the corresponding higher Hopf invariant. Let $S_{*}$ be the suspension homomorphism.

Theorem (4.6) (Sphere Theorem). Let $\phi \in \pi_{q}\left(S^{n}\right), n \geqq 2, v \in X^{S^{n}}$, and let $\zeta \in \pi_{n+1}\left(X, x_{0}\right)$. Then

$$
\phi_{v} v^{-1}(\zeta)=\zeta \circ S_{*} \phi+\sum_{0}^{\infty}(-1)^{p+1} \sigma_{p}\left(v_{*} \iota^{n}, \zeta\right) \circ S_{*} B_{p}(\phi) .
$$

In particular, the sphere theorem allows us to compute any homomorphism of the fundamental groups of the loop spaces $\pi_{1}\left(\Omega^{n} X, v\right) \rightarrow \pi_{1}\left(\Omega^{q} X, v f\right)$ induced by a map $f: S^{q} \rightarrow S^{n}$. 
Let $\beta, \phi, u, \xi$ be as above, and let $b:\left(S^{n}, s_{0}\right) \rightarrow\left(K, k_{0}\right)$ be a representative map for $\beta$. Then it follows from the definitions that

$$
(\beta \circ \phi)_{u}(\xi)=\phi_{u b}(u b) \mathfrak{\natural}^{-1} \beta_{u}(\xi) .
$$

Theorem (4.6), together with (4.7), yields

Corollary (4.8) (Composition Theorem).

$$
(\beta \circ \phi)_{u}(\xi)=\beta_{u}(\xi) \circ S_{*} \phi+\sum_{0}^{\infty}(-1)^{p+1} \sigma_{p}\left(u_{*} \beta, \beta_{u}(\xi)\right) \circ S_{*} B_{p}(\phi) .
$$

In particular, if $q<3 n-2$, then $B_{p}(\phi)=0$ for all $p>0$, and $B_{0}(\phi)=H(\phi)$, the generalized Hopf invariant. The formula then reduces to

$$
(\beta \circ \phi)_{u}(\xi)=\beta_{u}(\xi) \circ S_{*} \phi-\left[u_{*} \beta, \beta_{u}(\xi)\right] \circ S_{*} H(\phi) .
$$

Proof of (4.1). Let $a, b:\left(S^{q}, s_{0}\right) \rightarrow\left(K, k_{0}\right)$ represent $\alpha, \beta$ respectively. Denoting by $i=i^{q}:\left(I^{q}, \dot{I}^{q}\right) \rightarrow\left(S^{q}, s_{0}\right)$ a characteristic map for the cell $e^{q}=S^{q}-s_{0}$ as before, we can represent $\alpha+\beta$ by $c:\left(S^{q}, s_{0}\right) \rightarrow\left(K, k_{0}\right)$, defined by

$$
\begin{array}{rlrl}
c i\left(t_{1}, \cdots, t_{q}\right) & =a i\left(2 t_{1}, t_{2}, \cdots, t_{q}\right) & & \text { if } t_{1} \leqq 1 / 2 \\
& =b i\left(2 t_{1}-1, t_{2}, \cdots, t_{q}\right) & \text { if } t_{1} \geqq 1 / 2 .
\end{array}
$$

Let $F:\left(K \times I, k_{0} \times I\right) \rightarrow\left(X, x_{0}\right)$ represent $\xi \in \pi_{1}\left(X^{K}, u\right)$; then

$$
(\alpha+\beta)_{u}(\xi)=d\left(F(c \times 1), u^{b}(c \times 1)\right)\left(e^{q} \times e^{1}, s_{0} \times 0\right),
$$

where $e^{1}=I-\dot{I}$.

Let the subsets $I_{1}^{q}, I_{2}^{q} \subset I^{q}$ be determined by $t_{1} \leqq 1 / 2, t_{1} \geqq 1 / 2$, respectively, and define cells $\sigma_{1}, \sigma_{2} \subset I^{q} \times I$ as the interiors of $I_{1}^{q} \times I, I_{2}^{q} \times I$, with base points $p_{1}=0=(0, \cdots, 0), p_{2}=(1 / 2,0, \cdots, 0)$ respectively. Let $T$ be a path from 0 to $p_{2}$ given by $T(t)=(t / 2,0, \cdots, 0)$. Applying (3.5) to the separation element in (4.10), we obtain

$$
\begin{aligned}
(\alpha+\beta)_{u}(\xi)= & d\left(F(c i \times 1), u^{\prime}(c i \times 1)\right)\left(\sigma_{1}, p_{1}\right) \\
& +\left(u^{\prime}(c i \times 1) T\right)_{\sharp} d\left(F(c i \times 1), u^{\prime}(c i \times 1)\right)\left(\sigma_{2}, p_{2}\right) \\
= & \alpha_{u}(\xi)+(u c i T)_{\sharp \beta_{u}}(\xi) .
\end{aligned}
$$

If $q>1, u c i T$ is the constant path; if $q=1$, it represents $u_{*} \alpha$. This proves (4.1).

In order to prove (4.6) we need the following lemma:

Lemma (4.11). Let $\phi \in \pi_{q}\left(S^{n}, s_{0}\right), \zeta \in \pi_{n+1}\left(X, x_{0}\right)$. Then

$$
\phi_{x_{0}}\left(x_{0}\right)_{\natural}^{-1}(\zeta)=\zeta \circ S_{*} \phi .
$$

Proof. Let $F:\left(S^{n} \times I, s_{0} \times I\right) \rightarrow\left(X, x_{0}\right)$ represent $\left(x_{0}\right)_{4}^{-1} \zeta$ (so that $F\left(S^{n} \times \dot{I}\right)$ $=x_{0}$ ), and let $r: S^{k} \times I \rightarrow S^{k+1}$ be the identification map, of degree +1 , which pinches $S^{k} \times \dot{I} \cup s_{0} \times I$ to a point. Then the following diagram commutes, where $f$ represents $\phi$, and $F^{\prime}=F r^{-1}$ : 


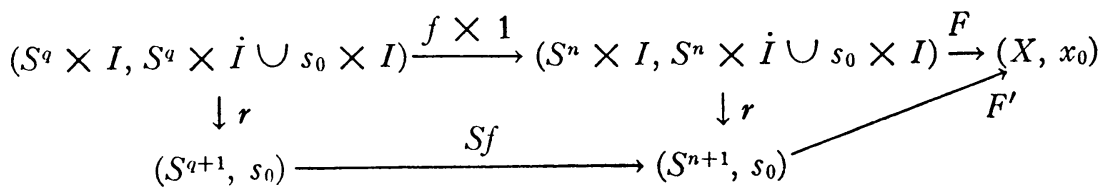

Clearly

$$
\zeta=\left(x_{0}\right)\left\{\{F\}=d\left(F, x_{0}^{b}\right)\left(e^{n} \times e^{1}, s_{0} \times 0\right)=\left\{F^{\prime}\right\} .\right.
$$

And similarly

$$
\begin{aligned}
\phi_{x_{0}}\{F\} & =d\left(F(f \times 1), x_{0}^{b}(f \times 1)\right)\left(e^{q} \times e^{1}, s_{0} \times 0\right) \\
& =\left\{F^{\prime}(S f)\right\}=\left\{F^{\prime}\right\} \circ S_{*} \phi=\zeta \circ S_{*} \phi .
\end{aligned}
$$

Proof of (4.6). Let $g: S^{n} \rightarrow S^{n} \bigvee S_{0}^{n}$ represent $\iota^{n}+\iota_{0}^{n}$, and let $u=v \bigvee x_{0}$ : $S^{n} \bigvee S_{0}^{n} \rightarrow X$. We identify

$$
\pi_{1}\left(X^{s^{n} \vee s_{0}^{n}}, u\right)=\pi_{1}\left(X^{s^{n}}, v\right)+\pi_{1}\left(X^{s_{0}^{n}}, x_{0}\right)
$$

in the natural way, so that elements of the group may be written $\left(v_{\natural}^{-1} \eta\right.$, $\left.\left(x_{0}\right)_{\natural}^{-1} \zeta\right)$, for $\eta, \zeta \in \pi_{n+1}\left(X, x_{0}\right)$; and we further abbreviate this notation to $(\eta, \zeta)$. It is easily verified that

$$
\iota_{u}^{n}(\eta, \zeta)=\eta, \quad\left(\begin{array}{c}
n \\
\iota_{0}
\end{array}\right)_{u}(\eta, \zeta)=\zeta .
$$

Then

$$
\begin{aligned}
\left(\left(\begin{array}{c}
n \\
\iota
\end{array}+\begin{array}{c}
n \\
\iota_{0}
\end{array}\right) \circ \phi\right)_{u}(0, \zeta) & =\phi_{u g}(u g)_{\text {घ }}^{-1}\left(\iota^{n}+{ }^{n}\right)_{0}(0, \zeta) \\
& =\phi_{u g}(u g)_{\text {ด }}^{-1} \zeta \text { by }(4.1),(4.12), \\
& =\phi_{v} v_{\natural}^{-1} \zeta \text { by }(2.6),(2.8) \text { since } v \simeq u g .
\end{aligned}
$$

On the other hand, we have the expansion of (4.5)

$$
\left(\iota^{n}+\iota_{0}^{n}\right) \circ \phi=\iota^{n} \circ \phi+\iota_{0}^{n} \circ \phi+\sum_{0}^{\infty} \omega_{i} \circ H_{i}(\phi),
$$

and we may apply the addition theorem to the left-hand side of (4.13) in this expanded form. Since $u_{*} \iota_{0}^{n}=0$, it follows from (4.4) that the expression

$$
\left(\omega_{i} \circ H_{i}(\phi)\right)_{u}(0, \zeta)=\left(H_{i}(\phi)\right)_{x_{0}}\left(x_{0}\right)_{\natural}^{-1}\left(\omega_{i}\right)_{u}(0, \zeta)
$$

is 0 if $\omega_{i}$ involves $\iota_{0}^{n}$ more than once. By definition $\left\{\sigma_{p}\left(\iota^{n}, \iota_{0}^{n}\right)\right\}, p=-1,0$, $1, \cdots$ consists of those basic products which involve $\iota_{0}^{n}$ only once. If $\omega_{i_{p}}=\sigma_{p}$, then writing $B_{p}(\phi)=H_{i_{p}}(\phi)$, we have by induction

$$
\begin{aligned}
& \sigma_{p}\left(\iota^{n}, \iota^{n} \iota_{0}\right)_{u}(0, \zeta)=-\left[u_{* \iota} \iota^{n}, \sigma_{p-1}\left(\iota^{n}, \iota^{n}\right)_{u}(0, \zeta)\right] \\
& =(-1)^{p+1} \sigma_{p}\left(u_{* \iota}{ }^{n},\left(\iota^{n}\right)_{u}(0, \zeta)\right) \\
& =(-1)^{p+1} \sigma_{p}\left(v_{* \iota}{ }^{n}, \zeta\right) \text {, }
\end{aligned}
$$


using (4.12) and the fact that $u_{*} \iota^{n}=v_{*} \iota^{n}$. Hence

$$
\begin{aligned}
\left(\sigma_{p}\left(\iota^{n}, \iota_{0}^{n}\right) \circ B_{p}(\phi)\right)_{u}(0, \zeta) & \left.=\left(B_{p}(\phi)\right)_{x_{0}}\left(x_{0}\right)\right)^{-1}\left((-1)^{p+1} \sigma_{p}\left(v_{*} \iota^{n}, \zeta\right)\right) \\
& =(-1)^{p+1} \sigma_{p}\left(v_{*}{ }^{n}, \zeta\right) \circ S_{*} B_{p}(\phi)
\end{aligned}
$$

by (4.11).

Applying the addition theorem to the left-hand side of (4.13), expanded as in (4.14), and using (4.12) and (4.15) to calculate the terms, we obtain the expression in Theorem (4.6).

5. Examples. Using the notation of (3.3), let $L=K \cup e^{q+1}$, where the class of the attaching map is $\alpha \in \pi_{q}\left(K, k_{0}\right)$, and let $u:\left(K, k_{0}\right) \rightarrow\left(X, x_{0}\right)$ have an extension over $L$. Then to classify the extensions of $u$, we must compute $\alpha_{u}$; and the theorems of the preceding section allow this to be done in certain cases. In particular, if we know the homomorphisms $\left(\delta_{t}\right)_{u}$ for certain elements $\delta_{t} \in \pi_{n_{t}}\left(K, k_{0}\right)$, then we may compute $\alpha_{u}$ for any $\alpha$ formed from the $\delta_{t}$ by the operations of addition, formation of Whitehead products, and composition with elements of homotopy groups of spheres. In the special case $K$ $=S^{n_{1}} \bigvee \cdots \bigvee S^{n_{r}}$, Hilton has shown that all elements of the homotopy groups of $K$ can be so formed from the generators $\iota^{n_{1}}, \cdots, \iota^{n_{r}}$.

As an example, let $K=S^{m} \bigvee S^{n}$, with $m \leqq n$, and suppose for simplicity that $q<3 m-2$. Let $\nu, \omega$ denote the classes of $v=u \mid S^{m}$, $w=u \mid S^{n}$ respectively. We identify

$$
\pi_{1}\left(X^{K}, u\right)=\pi_{1}\left(X^{s^{m}}, v\right)+\pi_{1}\left(X^{s^{n}}, w\right)
$$

in the natural way. Abbreviating $(\eta, \zeta)=\left(v_{\natural}^{-1} \eta, w_{\natural}^{-1} \zeta\right), \eta \in \pi_{m+1}\left(X, x_{0}\right)$, $\zeta \in \pi_{n+1}\left(X, x_{0}\right)$, we compute $\alpha_{u}(\eta, \zeta) \in \pi_{q+1}\left(X, x_{0}\right)$. Leaving aside the cases $m=1$ or $n=1$,

$$
\pi_{q}(K)=\pi_{q}\left(S^{m}\right)+\pi_{q}\left(S^{n}\right)+\left[\iota^{m}, \iota^{n}\right] \circ \pi_{q}\left(S^{m+n-1}\right) ;
$$

let $\alpha=\alpha_{1}+\alpha_{2}+\left[\iota^{m}, \iota^{n}\right] \circ \beta$, where $\alpha_{1} \in \pi_{q}\left(S^{m}\right), \alpha_{2} \in \pi_{q}\left(S^{n}\right), \beta \in \pi_{q}\left(S^{m+n-1}\right)$. Then

$$
\alpha_{u}(\eta, \zeta)=\left(\alpha_{1}\right)_{u}(\eta, \zeta)+\left(\alpha_{2}\right)_{u}(\eta, \zeta)+\left(\left[\iota^{m}, \iota^{n}\right] \circ \beta\right)_{u}(\eta, \zeta) .
$$

Now from (4.6)

$$
\begin{aligned}
& \left(\alpha_{1}\right)_{u}(\eta, \zeta)=\eta \circ S_{*} \alpha_{1}-[\nu, \eta] \circ S_{*} H\left(\alpha_{1}\right) \\
& \left(\alpha_{2}\right)_{u}(\eta, \zeta)=\zeta \circ S_{*} \alpha_{2}-[\omega, \zeta] \circ S_{*} H\left(\alpha_{2}\right)
\end{aligned}
$$

and from (4.2) and (4.8), since $\beta$ is a suspension,

$$
\left(\left[\iota^{m}, \iota^{n}\right] \circ \beta\right)_{u}(\eta, \zeta)=\left(-[\nu, \zeta]+(-1)^{n+1}[\eta, \omega]\right) \circ S_{*} \beta .
$$

This determines $\alpha_{u}(\eta, \zeta)$ as a sum of these expressions. If $m=n=1$, then $\alpha_{u}$ can be found by the addition theorem. If $m=1<n$, then $\alpha$ is a sum $\sum \xi_{i} \cdot \alpha_{i}$, $\xi_{i} \in \pi_{1}\left(S^{1}\right), \alpha_{i} \in \pi_{q}\left(S^{n}\right) . \alpha_{u}$ is then given by the addition theorem and (4.3). 
As a special case of the example, we consider maps $S_{1}^{n} \times S_{2}^{n} \rightarrow S^{n}, n \geqq 2$; here $\alpha=\left[\iota_{1}^{n}, \iota_{2}^{n}\right]$. If $v$, $w$ have degrees $p, q$ respectively, $p, q \neq 0$, we say that an extension of $u$ is of type $(p, q)$. The obstruction to such an extension is $u_{*}\left[\iota_{1}^{n}, \iota_{2}^{n}\right]=p q\left[\iota^{n}, \iota^{n}\right]$. Suppose that $u$ has an extension: then the homotopy classes of extensions are in 1-1 correspondence with $\pi_{2 n}\left(S^{n}\right) / \alpha_{u}\left(v_{\natural}^{-1} \pi_{n+1}\left(S^{n}\right)\right.$, $\left.w_{\natural}^{-1} \pi_{n+1}\left(S^{n}\right)\right)$. The subgroup contains only the elements $0, q\left[\iota^{n}, \eta\right], p\left[\iota^{n}, \eta\right]$, if $n \geqq 3$, where $\eta$ is the generator of $\pi_{n+1}\left(S^{n}\right)$. Now Hilton and Whitehead have shown [4] that $\left[\iota^{n}, \eta\right] \neq 0$ if and only if $n \equiv 1 \bmod 4$. Hence, using known results on Whitehead products,

EXAmple (5.1). There exist maps $S_{1}^{n} \times S_{2}^{n} \rightarrow S^{n}, n \geqq 2$, of type $(p, q), p, q \neq 0$, if and only if $n$ is odd, and either $p q$ is even or $\pi_{2 n+1}\left(S^{n+1}\right)$ has an element of Hopf invariant 1 . Suppose that $p, q$, and $n$ are such that maps do exist. Then the homotopy classes of such maps are in 1-1 correspondence with the elements of $\pi_{2 n}\left(S^{n}\right)$ if $p$ and $q$ are both even, or if $n \equiv-1 \bmod 4$; otherwise they are in 1-1 correspondence with the elements of

$$
\pi_{2 n}\left(S^{n}\right) /\left[\iota^{n}, \pi_{n+1}\left(S^{n}\right)\right]=\pi_{2 n}\left(S^{n}\right) / Z_{2} .
$$

Other examples are easily given; for instance

ExAmple (5.2). The identity map $S^{n} \rightarrow S^{n}$ always extends to maps $S^{n} \times S^{n-1} \rightarrow S^{n}$; the homotopy classes of extensions are in 1-1 correspondence with the elements of $\pi_{2 n-1}\left(S^{n}\right) /\left[\iota^{n}, \pi_{n}\left(S^{n}\right)\right] \approx S_{*} \pi_{2 n-1}\left(S^{n}\right)$.

EXAMPLE (5.3). Let $u$ be a map of $S^{1} \bigvee S^{1}$ into the real projective plane which is nontrivial on both circles. Then there are two homotopy classes rel $s_{0}$ of extensions of $u$ to $S^{1} \times S^{1}$.

6. An application: the group of homotopy equivalences. We shall outline an application of the above methods to the group of homotopy classes of homotopy equivalences of a space with itself, denoted $E q$.

Let $K$ be a 1 -connected CW complex, and let $K \cup_{e^{q+1}}$ be formed by attaching a cell $e^{q+1}, q>\operatorname{dim} K$, with $\alpha \in \pi_{q}(K)$ the class of the attaching map and $\bar{\alpha} \in \pi_{q+1}\left(K \cup e^{q+1}, K\right)$ the class of the characteristic map. Let

$$
i: K \subset K \cup e^{q+1}
$$

be the inclusion, and define a homomorphism

$$
d^{*}: i_{*} \pi_{q+1}(K) \rightarrow E q\left(K \cup e^{q+1}\right)
$$

as follows: $d^{*}(\beta)$ is the homotopy class of an extension $g$ of $i$ such that $d(g, 1)\left(e^{q+1}\right)=\beta$, where 1 denotes the identity map of $K \cup e^{q+1}$. Since $q>\operatorname{dim} K$ if $f$ is a homotopy equivalence of $K \cup e^{q+1}$, then $f_{*} \bar{\alpha}=\epsilon(f) \bar{\alpha}$, where $\epsilon(f)= \pm 1$. We also define homomorphisms

$$
j^{*}: E q\left(K \cup e^{q+1}\right) \rightarrow E q(K), \quad j_{0}^{*}: E q\left(K \cup e^{q+1}\right) \rightarrow E q\left(S^{q+1}\right),
$$

by $j^{*}\{f\}=\{f \mid K\}, j_{0}^{*}\{f\}=\epsilon(f) \iota^{q+1}$. 
THEOREM (6.1). The following sequences are exact:

$$
\begin{array}{ll}
i_{*} \pi_{q+1}(K) \stackrel{d^{*}}{\longrightarrow} E q\left(K \cup e^{q+1}\right) \stackrel{j^{*}}{\longrightarrow} E q(K), & \text { if } 2 \alpha \neq 0 ; \\
i_{*} \pi_{q+1}(K) \stackrel{d^{*}}{\longrightarrow} E q\left(K \cup e^{q+1}\right) \stackrel{j^{*}+j_{0}^{*}}{\longrightarrow} E q(K)+E q\left(S^{q+1}\right), & \text { if } 2 \alpha=0 .
\end{array}
$$

From Lemma 7 of [6] it follows that the image of $j^{*}$ is the set of classes $\{h\}$ such that $h_{*} \alpha= \pm \alpha$; denote this subgroup by $E q_{e}(K)$. The image of $j^{*}+j_{0}^{*}$ is then $E q_{e}(K)+E q\left(S^{q+1}\right)$, if $2 \alpha=0$. The kernel of $d^{*}$ is

$$
i_{*} \pi_{q+1}(K) \cap \alpha_{i} \pi_{1}\left(\left(K \cup e^{q+1}\right)^{K}, i\right)
$$

where the base point $k_{0} \in K$ is any point of $\dot{e}^{q+1}$. Methods were given in the previous sections for calculating $\alpha_{i}$ if $K$ is a bunch of spheres, so that in this case we can find $E q\left(K \cup e^{q+1}\right)$ up to extension.

The operations of $E q_{e}(K)$, or $E q_{e}(K)+E q\left(S^{q+1}\right)$, on $i_{*} \pi_{q+1}(K) / i_{*} \pi_{q+1}(K)$ $\bigcap \alpha_{i} \pi_{1}$ are given as follows: Let $\gamma \in \pi_{q+1}(K), \psi=\{h\} \in E q(K), \epsilon \iota^{q+1} \in E q\left(S^{q+1}\right)$. Then

(i) If $2 \alpha \neq 0$, then $\psi \cdot\left(i_{*} \gamma\right)=i_{*} h_{*} \gamma$;

(ii) If $2 \alpha=0$, then $\left(\psi, \epsilon \iota^{q+1}\right) \cdot\left(i_{*} \gamma\right)=\epsilon i_{*} h_{*} \gamma$.

The extension is not known to us, in general.

7. Further applications. In this section we shall show how the theory of $\S \S 2-4$ can be applied to obtain information about Whitehead products.

Theorem (7.1). If $\gamma \in \pi_{q}\left(S^{m}\right)$, then in $\pi_{q+n-1}\left(S^{m} \bigvee S^{n}\right)$ we have $\left[\iota^{m}{ }_{0} \gamma, \iota^{n}\right]$ $=\left[\iota^{m}, \iota^{n}\right] \circ S_{*}^{n-1} \gamma+\sum_{0}^{\infty}(-1)^{(p+1)(n+1)} \sigma_{p+1}\left(\iota^{m}, \iota^{n}\right) \circ S_{*}^{n-1} B_{p}(\gamma)$, for $m, n>1$, where $\sigma_{p+1}\left(\iota^{m}, \iota^{n}\right)$ and $B_{p}(\gamma)$ are defined as in (4.6).

Proof. Using the elementary relation

$$
\left[\eta, \iota^{1}\right]=\iota^{1} \cdot \eta-\eta, \quad \text { for } \eta \in \pi_{q}\left(S^{m}\right),
$$

to expand both sides of the identity $\left(\iota^{1} \cdot \iota^{m}\right) \circ \gamma=\iota^{1} \cdot\left(\iota^{m} \circ \gamma\right)$, we obtain

$$
\left(\left[\iota^{m}, \iota^{1}\right]+\iota^{m}\right) \circ \gamma=\left[\iota^{m} \circ \gamma, \iota^{1}\right]+\iota^{m} \circ \gamma .
$$

Now as shown in the addition theorem, if $u \in X^{K}, \xi \in \pi_{1}\left(X^{K}, u\right)$, then the transformation $(u, \xi): \pi_{q}\left(K, k_{0}\right) \rightarrow \pi_{q+1}\left(X, x_{0}\right)$ given by $(u, \xi) \alpha=\alpha_{u}(\xi)$ is a homomorphism for $q>1$. Taking $K=S^{m} \bigvee S^{1}, X=S^{m} \bigvee S^{2}, u$ such that $u_{*} \iota^{m}$ $=\iota^{m}, u_{*} \iota^{1}=0$, and $\xi$ such that $\iota_{u}^{m}(\xi)=0, \iota_{u}^{1}(\xi)=\iota^{2}$, and applying $(u, \xi)$ to both sides of (7.3), we obtain by use of the composition theorem

$$
\begin{aligned}
\left(-\left[\iota^{m}, \iota^{2}\right]+0\right) \circ S_{*} \gamma+\sum_{0}^{\infty}(-1)^{p+1} \sigma_{p}\left(0+\iota^{m},-\left[\iota^{m}, \iota^{2}\right]\right. & +0) \circ S_{*} B_{p}(\gamma) \\
& =-\left[\iota^{m} \circ \gamma, \iota^{2}\right]+0 ;
\end{aligned}
$$

using the definition of $\sigma_{p+1}\left(\iota^{m}, \iota^{n}\right)$, this yields the equation in (7.1) for the case $n=2$.

We can now prove (7.1) by induction on $n$. Suppose that (7.1) holds for 
$n$, and apply $(u, \xi)$ to both sides of the equation, with $K=S^{m} \vee S^{n}, X$ $=S^{m} \bigvee S^{n+1}, u$ such that $u_{*} \iota^{m}=\iota^{m}, u_{*} \iota^{n}=0$, and $\xi$ such that $\iota_{u}^{m}(\xi)=0, \iota_{u}^{n}(\xi)$ $=\iota^{n+1}$. We obtain

$$
\begin{aligned}
-\left[\iota^{m} \circ \gamma, \iota^{n+1}\right]= & -\left[\iota^{m}, \iota^{n+1}\right] \circ S_{* \gamma}^{n} \\
& +\sum_{0}^{\infty}(-1)^{(p+1)(n+1)}(-1)^{p+2} \sigma_{p+1}\left(\iota^{m}, \iota^{n+1}\right) \circ S_{*}^{n} B_{p}(\gamma)
\end{aligned}
$$

which yields the required equation for $n+1$. This proves (7.1).

Theorem (7.1) may be used as a universal example to derive

Corollary (7.4). If $\gamma \in \pi_{q}\left(S^{m}\right), \alpha \in \pi_{m}(X), \beta \in \pi_{n}(X), m, n>1$, then $[\alpha \circ \gamma, \beta]=[\alpha, \beta] \circ S_{*}^{n-1} \gamma+\sum_{0}^{\infty}(-1)^{(p+1)(n+1)} \sigma_{p+1}(\alpha, \beta) \circ S_{*}^{n-1} B_{p}(\gamma)$.

The corollary generalizes a formula of G. W. Whitehead [5, (3.59)] for the case in which $\gamma$ is a suspension.

As a further application, we give a simple inductive proof of the Jacobi identity for Whitehead products in $\pi_{p+q+r-2}\left(S^{p} \bigvee S^{q} \bigvee S^{r}\right)$ (cf. [3] et al.). With our conventions for the Whitehead product, the identity is given by

THEOREM (7.5).

$$
\begin{aligned}
(-1)^{(p+1) r}\left[\left[\iota^{p}, \iota^{q}\right], \iota^{r}\right]+(-1)^{(r+1) q}[ & {\left.\left[\iota^{r}, \iota^{p}\right], \iota^{q}\right] } \\
& +(-1)^{(q+1) p}\left[\left[\iota^{q}, \iota^{r}\right], \iota^{p}\right]=0, \quad p, q, r \geqq 2 .
\end{aligned}
$$

Proof. It is elementary that the following relation holds in $\pi_{2}\left(S^{2} \bigvee S_{0}^{2} \bigvee S^{1}\right)$ : $\iota^{1} \cdot\left[\iota^{2}, \iota_{0}^{2}\right]=\left[\iota^{1} \cdot \iota^{2}, \iota^{1} \cdot \iota_{0}^{2}\right]$. Expanding both sides by (7.2),

$$
\left[\left[\iota^{2}, \iota_{0}^{2}\right], \iota^{1}\right]+\left[\begin{array}{cc}
{ }^{2} & \iota_{0}^{2}
\end{array}\right]=\left[\left[\iota^{2}, \iota^{1}\right]+\iota^{2},\left[\begin{array}{cc}
2 & 1 \\
\iota_{0}, & \iota
\end{array}\right]+\iota_{0}^{2}\right] \text {. }
$$

Choosing $K=S^{2} \bigvee S_{0}^{2} \bigvee S^{1}, \quad X=S^{2} \bigvee S_{0}^{2} \bigvee S_{1}^{2} \quad u \in X^{K}$ such that $u_{*} \iota^{2}=\iota^{2}$, $u_{*} \iota_{0}^{2}=\iota_{0}^{2}, u_{*} \iota^{1}=0, \quad \xi \in \pi_{1}\left(X^{K}, u\right)$ such that $\iota_{u}^{2}(\xi)=\left(\iota_{0}^{2}\right)_{u}(\xi)=0, \iota_{u}^{1}(\xi)=\iota_{1}^{2}$, and applying $(u, \xi)$ to both sides of $(7.6)$ we obtain $-\left[\left[\iota^{2}, \iota_{0}^{2}\right], \iota_{1}^{2}\right]=\left[\iota_{0}^{2},\left[\iota_{0}^{2}, \iota_{1}^{2}\right]\right]$ $+\left[\left[\iota^{2}, \iota_{1}^{2}\right], \iota_{0}^{2}\right]$ which yields $(7.5)$ for $p=q=r=2$.

Suppose inductively that the identity of (7.5) holds for $p, q, r$. Taking $K=S^{p} \bigvee S^{q} \bigvee S^{r}, \quad X=S^{p} \bigvee S^{q} \bigvee S^{r+1}, u \in X^{K}$ such that $u_{*} \iota^{p}=\iota^{p}, u_{*} \iota^{q}=\iota^{q}$, $u_{*} \iota^{r}=0$, and $\xi \in \pi_{1}\left(X^{K}, u\right)$ such that $\iota_{u}^{p}(\xi)=\iota_{u}^{q}(\xi)=0, \iota_{u}^{r}(\xi)=\iota^{r+1}$, by applying $(u, \xi)$ to both sides of the equality in (7.5) we obtain the same equality with $r$ replaced by $r+1$. This proves (7.5).

The proof could equally well start with the relation $\iota^{1} \cdot\left[\iota_{0}^{1}, \iota_{1}^{1}\right]=\left[\iota^{1} \cdot \iota_{0}^{1}, \iota^{1} \cdot \iota_{1}^{1}\right]$ which can be verified purely formally.

Notice that if we apply homomorphisms $(u, \xi)$ to both sides of $(7.6)$ with $u$ and $\xi$ appropriately chosen to raise the dimensions of $\iota^{2}$ and $\iota_{0}^{2}$, but leave $\iota^{1}$ fixed, then we obtain a generalization of the Jacobi identity for the case in which one factor is of dimension 1 ; this can be written 


$$
\begin{aligned}
(-1)^{p+1}\left[\left[\iota^{p}, \iota^{q}\right], \iota^{1}\right]+ & {\left[\left[\iota^{1}, \iota^{p}\right], \iota^{q}\right] } \\
+ & (-1)^{(q+1) p}\left[\left[\iota^{q}, \iota^{1}\right], \iota^{p}\right]+\left[\left[\iota^{1}, \iota^{p}\right],\left[\iota^{q}, \iota^{1}\right]\right]=0 .
\end{aligned}
$$

Equation (7.7) also follows directly from the properties of the operation of $\pi_{1}$, in the manner of (7.6)

Theorem (7.5) is a universal example for the Jacobi identity in the homotopy groups of any space.

8. Proof of the product theorem. We shall now prove Theorem (4.2). As universal examples for $K, \gamma$, and $\delta$ we take $S^{m} \bigvee S^{n}, \iota^{m}$, and $\iota^{n}$ respectively. Then if $K, \gamma$, and $\delta$ are arbitrary, there is a map $h: S^{m} \bigvee S^{n} \rightarrow K$ such that $h_{*} \iota^{m}=\gamma$ and $h_{* \iota} \iota^{n}=\delta$. Since

$$
[\gamma, \delta]_{u}=\left(h_{*}\left[\iota^{m}, \iota^{n}\right]\right)_{u}=\left[\iota^{m}, \iota^{n}\right]_{u h} h^{*}
$$

and $\gamma_{u}=\iota_{u h}^{m} h^{*}, \delta_{u}=\iota_{u h}^{n} h^{*}$, one verifies immediately that Theorem (4.2) for the general case follows if we have proved it for the universal example.

Let $w: S^{m} \rightarrow X, \quad v: S^{n} \rightarrow X$ define $u=w \bigvee v: S^{m} \bigvee S^{n} \rightarrow X$; we identify $\pi_{1}\left(X s^{m} \vee s^{n}, u\right)=\pi_{1}\left(X s^{m}, w\right)+\pi_{1}\left(X s^{n}, v\right)$ under the natural isomorphism, so that there is a natural isomorphism $\left(w_{\natural}^{-1}, v_{\natural}^{-1}\right): \pi_{m+1}+\pi_{n+1} \rightarrow \pi_{1}\left(X s^{m} \vee s^{n}, u\right)$, where $\pi_{k}=\pi_{k}\left(X, x_{0}\right)$. Let

$$
\kappa=\left[\iota^{m}, \iota^{n}\right]_{u}\left(w^{-1}, v^{-1}\right): \pi_{m+1}+\pi_{n+1} \rightarrow \pi_{m+n} ;
$$

let $\omega, \nu$ denote $w_{*} \iota^{m}, v_{*} \iota^{n}$, and let $\lambda \in \pi_{m+1}, \rho \in \pi_{n+1}$. Then Theorem (4.2) for the universal example can be written

$$
\begin{array}{ll}
\text { (i) } \kappa(\lambda, \rho)=-[\omega, \rho]+(-1)^{n+1}[\lambda, \nu] & \text { if } m, n>1, \\
\text { (ii) } \kappa(\lambda, \rho)=-[\omega, \rho]+(-1)^{n+1}[\lambda, \omega \cdot \nu] & \text { if } m=1, n>1, \\
\text { (iii) } \kappa(\lambda, \rho)=-[\nu \cdot \omega, \rho]+(-1)^{n+1}[\lambda, \nu] & \text { if } m>1, n=1, \\
\text { (iv) } \kappa(\lambda, \rho)=-[\nu \cdot \omega, \rho]-\nu \cdot[\lambda,-(\omega \cdot \nu)] & \text { if } m=n=1 .
\end{array}
$$

We write the fundamental group additively, and shall first deduce (8.1) (iv) from the addition theorem. In this case $S^{m} \bigvee S^{n}=S^{1} \bigvee S^{1}$, and we set $\iota=\iota^{m}, \iota^{\prime}=\iota^{n}$. Then for

$$
\xi=\left(w_{\natural}^{-1} \lambda, v_{\natural}^{-1} \rho\right) \in \pi_{1}\left(X^{S^{1} \vee S^{1}}, u\right),
$$

it is clear that $\iota_{u}(\xi)=\lambda, \iota_{u}^{\prime}(\xi)=\rho$. Now $\left[\iota, \iota^{\prime}\right]=\left(\iota^{\prime}+\iota\right)-\left(\iota+\iota^{\prime}\right)$, and

$$
\left(\iota^{\prime}+\iota\right)_{u}(\xi)=\iota_{u}^{\prime}(\xi)+u_{*} \iota^{\prime} \cdot \iota_{u}(\xi)=\rho+\nu \cdot \lambda .
$$

Since

$$
\begin{aligned}
\left(-\left(\iota+\iota^{\prime}\right)+\left(\iota+\iota^{\prime}\right)\right)_{u}(\xi) & =\left(-\left(\iota+\iota^{\prime}\right)\right)_{u}(\xi)+(-(\omega+\nu)) \cdot\left(\iota+\iota^{\prime}\right)_{u}(\xi), \\
\left(-\left(\iota+\iota^{\prime}\right)\right)_{u}(\xi) & =-(-\nu-\omega) \cdot(\lambda+\omega \cdot \rho) .
\end{aligned}
$$

Therefore 


$$
\begin{aligned}
\kappa(\lambda, \rho) & =\left[\iota, \iota^{\prime}\right]_{u}(\xi)=\left(\left(\iota^{\prime}+\iota\right)-\left(\iota+\iota^{\prime}\right)\right)_{u}(\xi) \\
& =\rho+\nu \cdot \lambda-(\nu+\omega) \cdot((-\nu-\omega) \cdot(\lambda+\omega \cdot \rho)) \\
& =\nu \cdot(\lambda-(\omega-\nu-\omega) \cdot \lambda)+\rho-(\nu+\omega-\nu) \cdot \rho \\
& =-\nu \cdot[\lambda, \omega \cdot(-\nu)]-[\rho, \nu \cdot \omega] .
\end{aligned}
$$

This proves (8.1)(iv).

We can now suppose that $m+n>2$; if we prove that

$$
\begin{aligned}
\kappa(0, \rho) & =-[\omega, \rho] & & \text { if } n>1, \\
& =-[\nu \cdot \omega, \rho] & & \text { if } n=1,
\end{aligned}
$$

it will follow that

$$
\begin{aligned}
\kappa(\lambda, 0) & =\left[\iota^{m}, \iota^{n}\right]_{u}\left(w_{\natural}^{-1} \lambda, v_{\natural}^{-1} 0\right)=\left((-1)^{m n}\left[\iota^{n}, \iota^{m}\right]\right)_{u}\left(w_{\natural}^{-1} \lambda, v_{\natural}{ }^{-1} 0\right) \\
& =(-1)^{m n+1}[\nu, \lambda] \text { or }(-1)^{n+1}[\omega \cdot \nu, \lambda] \\
& =(-1)^{n+1}[\lambda, \nu] \text { or }(-1)^{n+1}[\lambda, \omega \cdot \nu]
\end{aligned}
$$

according as $m>1$ or $m=1$. Then (8.3) implies that $\kappa(\lambda, \rho)=\kappa(\lambda, 0)+\kappa(0, \rho)$ is given by (8.1)(i), (ii), or (iii), and we need only prove (8.3).

Consider the case $\left(X, x_{0}\right)=\left(S^{m} \bigvee S^{n+1}, s_{0}\right), w=j^{m}$, the identity map of $S^{m}$, $v=s_{0}, \rho=\iota^{n+1}$, where $m, n \geqq 1$ and $m+n>2$; we prove by considering representative maps that

Lemma (8.4). In this case $\kappa\left(0, \iota^{n+1}\right)=-\left[\iota^{m}, \iota^{n+1}\right]$.

Proof. $\left[\iota^{m}, \iota^{n}\right]$ is represented by $p: \dot{I}^{m+n}=\left(I^{m} \times I^{n}\right)^{\cdot} \rightarrow S^{m} \bigvee S^{n}$,

$$
\begin{aligned}
p\left(y, y^{\prime}\right) & =i^{m}(y) & & \text { if } y \in I^{m}, y^{\prime} \in \dot{I}^{n}, \\
& =i^{n}\left(y^{\prime}\right) & & \text { if } y \in \dot{I}^{m}, y^{\prime} \in I^{n} .
\end{aligned}
$$

Define maps $E, F:\left(\left(S^{m} \bigvee S^{n}\right) \times I, s_{0} \times I\right) \rightarrow\left(S^{m} \bigvee S^{n+1}, s_{0}\right)$ by

$$
\begin{array}{rlrl}
E(z, t) & =z, & z \in S^{m}, t \in I, \\
& =s_{0}, & & z \in S^{n} ; \\
F(z, t) & =z, & z \in S^{m}, \\
& =i^{n+1}\left(\left(i^{n}\right)^{-1}(z), t\right), & & z \in S^{n} .
\end{array}
$$

Then $F(p \times 1), \quad E(p \times 1):\left(\dot{I}^{m+n} \times I, \quad 0 \times I\right) \rightarrow\left(S^{m} \bigvee S^{n+1}, \quad s_{0}\right)$ agree on $\dot{I}^{m+n}$ $\times \dot{I} \cup 0 \times I$; and since $F$ represents $\left(\left(j^{m}\right)_{\natural}^{-1} 0, s_{04}^{-1} \iota^{n+1}\right) \in \pi_{1}\left(X^{s^{m} \vee s^{n}}, u\right)$, we have

$$
\kappa\left(0, \iota^{n+1}\right)=d\left(F(p \times 1),(E(p \times 1))\left(\left(\dot{I}^{m+n}-0\right) \times I, 0 \times 0\right) .\right.
$$

Extend $F(p \times 1), E(p \times 1)$ over $\dot{I}^{m+n+1}=\dot{I}^{m+n} \times I \cup I^{m+n} \times \dot{I}$ to $\bar{F}, \bar{E}$ respectively, as follows: for $y \in I^{m}, y^{\prime} \in I^{n}, t \in \dot{I}$ define

$$
\begin{aligned}
\bar{F}\left(y, y^{\prime}, t\right) & =i^{m}(y) & & \text { if } y^{\prime} \in \dot{I}^{n}, \\
& =i^{n+1}\left(y^{\prime}, t\right)=s_{0} & & \text { if } y \in \dot{I}^{m} ;
\end{aligned}
$$


and the same for $\bar{E} . \bar{F}$ and $\bar{E}$ are readily seen to be the canonical maps representing $\left[\iota^{m}, \iota^{n+1}\right]$ and $\left[\iota^{m}, 0\right]$ respectively; also, $\bar{F}$ and $\bar{E}$ agree on $I^{m+n} \times \dot{I} \cup 0$ $\times I$. Setting $e^{m+n+1}=\dot{I}^{m+n+1}-0$, and applying Lemma (3.5) as in the proof of (3.2), it follows that $d(F(p \times 1), E(p \times 1))=d\left(\bar{F}\left|\dot{I}^{m+n} \times I, \bar{E}\right| \dot{I}^{m+n} \times I\right)$ on $\left(\left(\dot{I}^{m+n}-0\right) \times I, 0 \times 0\right)$ is equal to $-d(\bar{F}, \bar{E})\left(e^{m+n+1}, 0\right)$. Thus

$\kappa\left(0, \iota^{n+1}\right)=-d(\bar{F}, \bar{E})\left(e^{m+n+1}, 0\right)=-(\{\bar{F}\}-\{\bar{E}\})=-\{\bar{F}\}=-\left[\iota^{m}, \iota^{n+1}\right]$

which proves (8.4).

The space $S^{m} \bigvee S^{n+1}$ in (8.4) is a universal example for the case $v=x_{0}$; for, given any $\left(X, x_{0}\right), w, \rho$, there exists $g:\left(S^{m} \bigvee S^{n}, s_{0}\right) \rightarrow\left(X, x_{0}\right)$ such that $g \mid\left(S^{m}, s_{0}\right)=w$ and $\left\{g \mid S^{n+1}\right\}=\rho$.

Corollary (8.5). $\kappa(0, \rho)=-[\omega, \rho]$ if $v=x_{0}$, with $X, w, \rho$ arbitrary.

Now let all of $X, w, v, \rho$ be arbitrary. Define $h=j^{m} \bigvee h^{\prime}:\left(S^{m} \bigvee S^{n}, s_{0}\right)$ $\rightarrow\left(S^{m} \bigvee S_{1}^{n} \bigvee S_{2}^{n}, s_{0}\right)$, where $h^{\prime}: S^{n} \rightarrow S_{1}^{n} \bigvee S_{2}^{n}$ is such that $h_{*}^{\prime} \iota^{n}=\iota_{1}^{n}+\iota_{2}^{n}$. Let

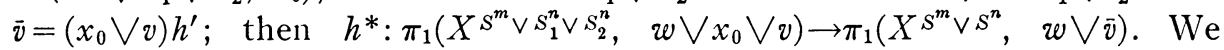
identify the second group with $\pi_{1}\left(X s^{m}, w\right)+\pi_{1}\left(X^{s^{n}}, \bar{v}\right)$, and treat the first similarly. Then it is clear from the definition of $\xi$ as a separation element, and from the definition of $h^{*}$, that

$$
h^{*}\left(w_{\natural}^{-1} \lambda, x_{0}^{-1} \rho_{1}, v_{\natural}^{-1} \rho_{2}\right)=\left(w_{\natural}^{-1} \lambda, \bar{v}_{\natural}^{-1}\left(\rho_{1}+\rho_{2}\right)\right) \text {. }
$$

Let $M$ be a homotopy rel $S^{m}$ from $w \bigvee \bar{v}$ to $u=w \bigvee v$; under the above identification $M_{\sharp}=\left(\left(M \mid S^{m}\right) \sharp,\left(M \mid S^{n}\right) \sharp\right)$. Since $v_{\natural}=\left(M \mid S^{n}\right) \sharp \bar{\nu}_{\sharp}$ by $(2.6)$, and $\left(M \mid S^{m}\right) \sharp$ is the identity,

$$
M \#\left(w_{\natural}^{-1} \lambda, \bar{v}_{\natural}^{-1}\left(\rho_{1}+\rho_{2}\right)\right)=\left(w_{\natural}^{-1} \lambda, v_{\natural}^{-1}\left(\rho_{1}+\rho_{2}\right)\right) .
$$

Setting $r=w \bigvee x_{0} \bigvee v$,

$$
\left[\begin{array}{cc}
\iota^{m} & \iota_{1}^{n}+\iota_{2}{ }^{n}
\end{array}\right]_{r}=\left(h_{*}\left[\iota^{m}, \iota^{n}\right]\right)_{r}=\left[\iota^{m}, \iota^{n}\right]_{r h} h^{*}=\left[\iota^{m}, \iota^{n}\right]_{u} M_{\sharp} h^{*}
$$

and hence

$$
\begin{aligned}
\kappa(0, \rho) & =\left[\iota^{m}, \iota^{n}\right]_{u}\left(w_{\natural}^{-1} 0, v_{\natural}^{-1} \rho\right) \\
& =\left[\iota^{m}, \iota^{n}\right]_{u} M \sharp h^{*}\left(w_{\natural}^{-1} 0, x_{0 \natural}^{-1} \rho, v_{\natural}^{-1} 0\right) \\
& =\left[\iota^{m}, \iota_{1}^{n}+\iota_{\iota}^{n}\right]_{r}\left(w_{\natural}^{-1} 0, x_{0 \natural}^{-1} \rho, v_{\natural}^{-1} 0\right) .
\end{aligned}
$$

If $m \geqq 1, n>1$, then it follows from the addition theorem that $\left[\iota^{m}, \iota_{1}^{n}+\iota_{2}^{n}\right]_{r}$ $=\left[\iota^{m}, \iota_{1}^{n}\right]_{r}+\left[\iota^{m}, \iota_{2}^{n}\right]_{r}$. The first term yields $\left[\iota^{m}, \iota_{1}^{n}\right]_{r}\left(w_{\natural}^{-1} 0, x_{0 \uparrow}^{-1} \rho, v_{\natural}^{-1} 0\right)=-[\omega, \rho]$ by $(8.5)$, while the second term yields 0 . Hence

$$
\kappa(0, \rho)=-[\omega, \rho] \quad \text { for } m \geqq 1, n>1 .
$$

If $m>1, n=1$, then

$$
\left[\iota^{m}, \iota_{1}^{n}+\iota_{2}^{n}\right]=\left(\iota_{1}^{n}+\iota_{2}^{n}\right) \cdot \iota^{m}-\iota^{m}=\left[\begin{array}{cc}
n & \iota_{2} \cdot \iota^{m}, \iota_{1}^{n}
\end{array}\right]+\left[\begin{array}{cc}
\iota^{m}, & \iota_{2}
\end{array}\right] .
$$


Applying the addition theorem, and noting that the second term again gives 0 ,

$$
\left[\iota^{m}, \iota_{1}^{n}+\iota_{2}^{n}\right]_{r}\left(w_{\natural}^{-1} 0, x_{0 \natural}^{-1} \rho, v_{\natural}^{-1} 0\right)=\left[\iota_{2}^{n} \cdot \iota^{m}, \iota_{1}^{n}\right]_{r}\left(w_{\natural}^{-1} 0, x_{0 \natural}^{-1} \rho, v_{\natural}^{-1} 0\right) .
$$

Let $k=l \bigvee j_{1}^{n}:\left(S^{m} \bigvee S^{n}, s_{0}\right) \rightarrow\left(S^{m} \bigvee S_{1}^{n} \bigvee S_{2}^{n}, s_{0}\right)$, where $l$ represents $\iota_{2}^{n} \cdot \iota^{m}$, so that $r k$ represents $\nu \cdot \omega$ on $S^{m}$ and 0 on $S^{n}$. Then $k^{*}: \pi_{1}\left(X S^{m} \vee S_{1}^{n} \vee S_{2, r}^{n}\right)$ $\rightarrow \pi_{1}\left(X s^{m} \vee s^{n}, r k\right)$ is clearly such that

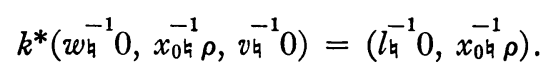

Since $k_{*}\left[\iota^{m}, \iota^{n}\right]=\left[\iota_{2}^{n} \cdot \iota^{m}, \iota_{1}^{n}\right]$,

$$
\begin{aligned}
& {\left[\iota_{2}^{n} \cdot \iota^{m}, \iota_{1}^{n}\right]_{r}\left(w_{\natural}^{-1} 0, x_{0 \natural}^{-1} \rho, v_{\natural}^{-1} 0\right)=\left[\iota^{m}, \iota^{n}\right]_{r k} k^{*}\left(w_{\natural}^{-1} 0, x_{0 \natural}^{-1} \rho, v_{\natural}^{-1} 0\right)} \\
& =\left[\iota^{m}, \iota^{n}\right]_{r k}\left(l_{\natural}^{-1} 0, x_{0 \natural}^{-1} \rho\right)=-[\nu \cdot \omega, \rho] \text { by }(8.5) \text {. }
\end{aligned}
$$

Equations (8.6), (8.8), and (8.9) yield

$$
\kappa(0, \rho)=-[\nu \cdot \omega, \rho] \quad \text { if } m>1, n=1 .
$$

Equations (8.7) and (8.10) together prove (8.3), and hence Theorem (4.2).

\section{Appendix. Separation elements}

Let $I^{n}$ be the subset of Euclidean $n$-space consisting of $n$-tuples of real numbers $\left(y_{1}, \cdots, y_{n}\right), 0 \leqq y_{i} \leqq 1$, oriented by the generator of $H_{n}\left(I^{n}, \dot{I}^{n}\right)$ represented by the identity map of $I^{n}$ in the cubical singular theory. Let $J^{n-1}$ be the closure of the subset of $\dot{I}^{n}$ for which $y_{n}<1$, and let $I_{1}^{n-1}$ be the subset of $\dot{I}^{n}$ for which $y_{n}=1$. If $x_{0} \in A \subset X$, then elements of $\pi_{n}\left(X, A, x_{0}\right)$ are represented by maps $f:\left(I^{n}, \dot{I}^{n}, J^{n-1}\right) \rightarrow\left(X, A, x_{0}\right)$, and the boundary operator

$$
\partial: \pi_{n}\left(X, A, x_{0}\right) \rightarrow \pi_{n-1}\left(A, x_{0}, x_{0}\right)=\pi_{n-1}\left(A, x_{0}\right)
$$

is defined by $\partial\{f\}=\left\{f \mid I_{1}^{n-1}\right\}$. If we identify $\left(S^{n-1}, s_{0}\right)=\left(\dot{I}^{n}, 0\right)$, where $0=(0, \cdots, 0)$, then the specification of the boundary operator determines an orientation of $S^{n-1}$ (cf. $[7, \S 4]$ ). It is to be noted that this is not the orientation given by the homology boundary.

Let $h_{t}:\left(I^{n}, \dot{I}^{n}, J^{n-1}, 0\right) \rightarrow\left(I^{n}, \dot{I}^{n}, J^{n-1}, 0\right)$ be a homotopy such that $h_{0}$ =identity, $h_{1}\left(J^{n-1}\right)=0 . h_{t}$ determines a $1-1$ correspondence between the sets of homotopy classes of maps $g:\left(I^{n}, \dot{I}^{n}, 0\right) \rightarrow\left(X, A, x_{0}\right)$ and the elements of $\pi_{n}\left(X, A, x_{0}\right)$ by $\{g\} \rightarrow\left\{g h_{1}\right\}$, and similarly between the homotopy classes of maps $g^{\prime}:\left(\dot{I}^{n}, 0\right) \rightarrow\left(A, x_{0}\right)$ and the elements of $\pi_{n-1}\left(A, x_{0}\right)$. Using this correspondence, we may represent elements of $\pi_{n}\left(X, A, x_{0}\right)$ and $\pi_{n-1}\left(A, x_{0}\right)$ by maps of $\left(I^{n}, \dot{I}^{n}, 0\right)$ and $\left(\dot{I}^{n}, 0\right)$ respectively.

We define separation elements as follows (cf. [1] for the original definition). Let $K$ be a CW complex $\left({ }^{2}\right)$ and let $\sigma \in K$ be an $n$-cell with characteristic map $c_{\sigma}:\left(I^{n}, \dot{I}^{n}, 0\right) \rightarrow\left(\bar{\sigma}, \dot{\sigma}, p_{\sigma}\right)$, where $p_{\sigma} \in \dot{\sigma}$ is a point. If $f, g:\left(\bar{\sigma}, p_{\sigma}\right) \rightarrow\left(X, x_{0}\right)$

(2) A fixed choice of characteristic map for each cell is implied in the definition of a $\mathrm{CW}$ complex. 
agree on $\dot{\sigma}$, they determine a separation element $d(f, g)\left(\sigma, p_{\sigma}\right) \in \pi_{n}\left(X, x_{0}\right)$, represented by $F:\left(\dot{I}^{n+1}, 0\right) \rightarrow\left(X, x_{0}\right)$,

$$
F\left(y_{1}, \cdots, y_{n+1}\right)=\left\{\begin{array}{lr}
f c_{\sigma}\left(y_{1}, \cdots, y_{n}\right) & \text { if } y_{n+1}=1, \\
f c_{\sigma}\left(y_{1}, \cdots, y_{n}\right)=g c_{\sigma}\left(y_{1}, \cdots, y_{n}\right) & 0<y_{n+1}<1, \\
g c_{\sigma}\left(y_{1}, \cdots, y_{n}\right) & \text { if } y_{n+1}=0 .
\end{array}\right.
$$

Thus $d(f, g)\left(\sigma, p_{\sigma}\right)=d\left(f c_{\sigma}, g c_{\sigma}\right)\left(I^{n}, 0\right)$ (we shall not bother to distinguish between the open and the closed cell, provided this causes no confusion).

It follows from the orientation convention that if $f(\dot{\sigma})=x_{0}, g(\bar{\sigma})=x_{0}$, then $d(f, g)\left(\sigma, p_{\sigma}\right)=\left\{f c_{\sigma}\right\}$.

\section{REFERENCES}

1. S. Eilenberg, Cohomology and continuous mappings, Ann. of Math. vol. 41 (1940) pp. 231-251.

2. R. H. Fox, On topologies for function spaces, Bull. Amer. Math. Soc. vol. 51 (1945) pp. 429-432.

3. P. J. Hilton, On the homotopy groups of the union of spheres, J. London Math. Soc. vol. 30 (1955) pp. 154-171.

4. P. J. Hilton and J. H. C. Whitehead, Note on the Whitehead product, Ann. of Math. vol. 58 (1953) pp. 429-442.

5. G. W. Whitehead, A generalization of the Hopf invariant, Ann. of Math. vol. 51 (1950) pp. 192-237.

6. J. H. C. Whitehead, On simply-connected, 4-dimensional polyhedra, Comment. Math. Helv. vol. 22 (1949) pp. 48-92.

7. - On certain theorems of G. W. Whitehead, Ann. of Math. vol. 58 (1953) pp. 418428. 428.

8. - On adding relations to homotopy groups, Ann. of Math. vol. 42 (1941) pp. 409-

9. J. R. Jackson, Spaces of mappings on topological products with applications to homotopy theory, Proc. Amer. Math. Soc. vol. 3 (1952) pp. 327-333.

Brown UNIVERSITY, PRovidence, R. I.

Brasenose College,

OXFORD, ENGLAND 